\title{
Sound propagation in suspensions of solid spheres
}

\author{
Ling Ye, ${ }^{*}$ Jing Liu, ${ }^{\dagger}$ Ping Sheng, and D. A. Weitz \\ Exxon Research and Engineering Co., Route 22E, Annandale, New Jersey 08801
}

(Received 9 February 1993)

\begin{abstract}
We measure the dispersion of the longitudinal sound waves in a suspension of solid spheres using Brillouin scattering. We find two distinct propagating longitudinal modes when the wavelength of the sound becomes comparable to the sphere diameter. The higher-frequency mode has a velocity intermediate between those of the pure solid and pure liquid phases, and its velocity increases with increasing solid volume fraction. The dispersion curve of this mode has distinct gaps, and the group velocity goes to zero near these gaps. We interpret this mode as a compressional excitation which propagates through both the liquid and the solid, as expected for a composite medium. The gaps in the dispersion curve result from the very large scattering of the excitation by the spheres, and occur at frequencies where the scattering from a single, isolated sphere is predicted to be a maximim due to a resonance in the sphere. By contrast, the lower-frequency mode has a velocity that is less than those in either the pure solid or the pure fluid. We interpret this mode as a surface acoustic excitation, which propagates between adjacent spheres by means of the exponentially decaying portion of the excitation in the fluid at the surface of the spheres. A summary of a theoretical treatment is also presented.
\end{abstract}

PACS number(s): 82.70.Dd, 62.30. $+\mathrm{d}$, 68.45.Nj, 78.35. $+\mathrm{c}$

\section{INTRODUCTION}

The propagation of sound through random media comprised of a composite of solid and fluid has long been a subject of great interest and importance. Its importance stems in part from the use of acoustic probes for nondestructive testing and for seismic exploration. In both cases, random, fluid-filled media are frequently encountered, and knowledge of the propagation of sound through these materials is essential for proper interpretation of the data. The propagation of sound through these materials depends sensitively on the connectivity and structure of the solid grains, and, depending on the nature of the medium, can exhibit a rich variety of fascinating behavior. One of the simplest, yet most fundamental geometries is that of a suspension of solid spheres immersed in a fluid. The study of such a system dates back to the work of Lord Rayleigh, who studied the scattering of sound waves from isolated spheres in a fluid [1,2]. More recently, suspensions of spheres at higher concentration have been investigated in the long-wavelength limit, $\lambda \gg d$, where $\lambda$ is the wavelength of the sound and $d$ the diameter of the spheres $[3,4]$. At these high concentrations, multiple scattering of the sound becomes important, and theoretical treatments of the behavior have followed an approach which explicitly considers the multiple scattering of waves in the fluid by the solid spheres [4]. The predicted results are in good agreement with extensive experimental results obtained for the propagation of ultrasonic waves through suspensions of uniform glass beads in a fluid. Since the continuous phase is a fluid and cannot support shear, only a single longitudinal mode can propagate in the limit of $\lambda \gg d$ [5]. The same behavior was long expected to persist to shorter wavelengths, where $\lambda \sim d$. In this regime, the scattering from individual spheres can excite internal resonances within the spheres, and some recent experiments on the propagation of sound through curing epoxies have been interpreted in terms of localization of sound [6]. However, a more carefully controlled study of sound propagation through a suspension of hard-sphere colloidal particles identified a new propagating longitudinal acoustic excitation when $\lambda \sim d[7,8]$. This mode was interpreted as surface excitation which propagated coherently between adjacent spheres. At even shorter wavelengths, this excitation reverts to a Stoneley wave [9], a propagating excitation confined to the interface between an elastic solid and a fluid. In this paper, we present a more detailed account of the behavior of sound propagation through suspensions of hard-sphere colloidal particles, and discuss the dispersion of the phonons in this system as the sound wavelength $\lambda$ is varied from much larger than the diameter of the spheres, $d$, to comparable to $d$, to much shorter than $d$.

We study the propagation of sound through colloidal suspensions interacting solely through the excluded volume interaction, or hard-sphere colloids. The characteristics of such a colloidal suspension are highly controllable, as the particle size, solid volume fraction, acoustic wavelength, and acoustic frequency can all be independently varied. The colloidal particles used in this work are suspended in a fluid that has the same index of refraction for light as do the particles themselves, thus eliminating any multiple scattering of light. This enables us to use light scattering techniques to probe the structural and acoustic properties of the suspension. We use static light scattering to determine the structural correlations of the spheres by measuring the static structure factor $S(q)$, where the scattering wave vector is $q=(4 \pi n / \lambda) \sin \theta / 2$, with $n$ the index of refraction and $\theta$ the scattering angle. We use Brillouin scattering to measure the frequency of the thermally excited sound modes within the suspension. A peak is observed in the Bril- 
louin spectrum at a frequency corresponding to a propagating acoustic excitation whose wavelength matches the inverse of the scattering wave vector. By varying the scattering angle, we can vary $q$ and hence the size of the acoustic wavelength relative to the sphere diameter. We can thereby determine the full dispersion curves of phonons in a suspension of hard-sphere colloids. We measure these dispersion curves for different volume fractions of the solids, and for particles of different diameters.

\section{EXPERIMENT}

The colloidal particles are monodisperse spheres of polymethylmethacrylate (PMMA), coated by a thin layer of grafted polymer with a thickness of about $15 \mathrm{~nm}$, much smaller than the diameter of the particles themselves. The polymer coating sterically stabilizes the particles, and ensures that the interaction between them is very well approximated as that of hard spheres [10]. These colloids exhibit a very rich phase behavior as the effective particle volume fraction (particle volume fraction including the grafted polymer layer) $\phi_{e}$ is changed. At low $\phi_{e}$, the colloidal particles behave as a fluid, with short-range correlations between the colloidal particles that are fluidlike. At larger volume fractions, $\phi_{e}>0.49$, the colloidal particles can order to form a colloidal crystal. For volume fractions between $\phi_{e} \approx 0.49$ and $\phi_{e} \approx 0.53$, the colloidal liquid and crystal phases coexist, while for $\phi_{e}$ greater than 0.53 , the equilibrium phase is purely crystalline. However, once the volume fraction becomes greater than about 0.56 , there is no longer sufficient room for the particles to rearrange themselves to attain long-range crystalline order, and the structure remains a disordered glass. In this work, we do not study the effects of crystalline order on the propagation of sound, but rather restrict our attention to the case of the disordered structures formed when the sample is initially tumbled to form a well mixed state.

The colloids were suspended in a mixture of dodecane and carbon disulfide. The index of refraction of PMMA is 1.51 , while that of dodecane is 1.42 and that of carbon disulfide is 1.62 . Thus by choosing the appropriate mixture of the two suspending fluids, the suspensions could be perfectly index matched, eliminating any multiple scattering of the light. Nevertheless, the Rayleigh peak was invariably about four orders of magnitude more intense than the Brillouin peak, due to the remnant scattering from the spheres. Thus the Brillouin scattering was resolved by using a Fabry-Pérot interferometer operated in a five-pass configuration, which provided sufficient contrast and resolution to observe the spectra. The samples were held in curvets with four flat surfaces, $1 \mathrm{~cm}$ on a side. The tops were sealed with Teflon stoppers to prevent the evaporation of the solvent.

The laser source for the Brillouin scattering was either an $\mathrm{Ar}^{+}$laser operated at $5145 \AA$, or a Kr ${ }^{+}$laser, operated at $6471 \AA$. In either case, an internal étalon was used to force the laser to operate on a single longitudinal mode. The spectra at a given $q$ were found to be independent of the laser wavelength. The colloids exhibit strong self-focusing of the incident laser, which is more pro- nounced in the green than in the red. Thus the incident power was kept sufficiently low to avoid any deleterious effects due to the self-focusing. Moreover, we ascertained that the spectra acquired were independent of the incident laser power. Thus the incident power was maintained below about $100 \mathrm{~mW}$, focused to a spot size of about $100 \mu \mathrm{m}$ in diameter. The scattered light was imaged onto a $150-\mu \mathrm{m}$-diam pinhole to improve the resolution and reduce parasitic scattering, and then collimated into the Fabry-Pérot interferometer. The finesse of the Fabry-Pérot interferometer was about 50, while the contrast was about $10^{10}$. The output of the Fabry-Pérot interferometer was detected using a cooled photomultiplier tube, operated in the standard photon counting configuration. The alignment of the Fabry-Pérot interferometer was actively stabilized, and spectra were typically collected for roughly $1 \mathrm{~h}$ to obtain data of sufficient statistical accuracy.

We can determine the phase velocity of the acoustic excitations from the frequencies of the peaks in the Brillouin spectra, $v=\omega / q$. We measure the polarized Brillouin scattering which is sensitive only to longitudinal excitations in the system. By varying the scattering angle, we can vary the scattering wave vector $q$, enabling us to measure the dispersion curve for the longitudinal phonons in the hard-sphere system. Our measurements are made at scattering angles ranging from $9^{\circ}$ to $170^{\circ}$, corresponding to $q$ varying from 0.002 to $0.04 \mathrm{~nm}^{-1}$, using the two laser lines. An important physical parameter in determining the behavior of this system is $q d$, or the ratio of the acoustic wavelength, determined by the scattering wave vector, to the diameter of the spheres, $d$, where we consider the diameter only of the core of the spheres, without the grafted polymer layer. To extend the accessible range of $q d$, we use two sets of spheres, with different diameters. The first set has a core diameter of $340 \mathrm{~nm}$, so that their diameter with the grafted polymer coating is $370 \mathrm{~nm}$. These spheres allow data to be collected for $q d$ ranging from about 1 to 14 . They are particularly well suited for collecting data near the peak in the static structure factor, $q d \approx 2 \pi$, where the acoustic wavelength and the sphere diameter are equal. The second set of spheres has a core diameter of $650 \mathrm{~nm}$ so that their diameter including the grafted polymer layer is $680 \mathrm{~nm}$. With these spheres, we can collect data for $q d$ ranging from about 2 to 25. Thus these spheres are most suitable for studying the behavior as $q d$ becomes much larger, so the acoustic wavelength becomes smaller than the sphere diameter.

To fully determine the behavior of this system, we obtain data for different volume fractions of the spheres. To measure the volume fraction, the samples are gently centrifuged until the colloids settle at the bottom of the containers. We assume that the sediment consists of randomly close-packed spheres. However, the packing of the spheres is determined by their outer radii, including the grafted polymer layer. Thus we assume that the effective volume fraction of the sediment is $\phi_{e}=0.64$, and calculate the true solid volume fraction $\phi$ by correcting for the thickness of the grafter polymer layer, assuming its thickness to be $15 \mathrm{~nm}$. The volume fraction of the close-packed sediment is the highest value of $\phi$ that we 
study. To study lower values of $\phi$, we measure the height of the sediment and adjust the height of the supernatant to obtain the desired volume fraction when the sample is remixed by tumbling. We estimate that the error in determining the volume fraction is about $\pm 5 \%$, due primarily to the uncertainty in measuring the heights. We note that, because of the grafted polymer layer, the cores of the colloidal particles form a disconnected system of solid spheres, even at the highest volume fractions measured. Finally, we ensure that none of the samples are allowed to settle and form colloidal crystals, so that there is no long-range positional order between the particles used in the experiments reported here.

\section{RESULTS}

The average structure in the colloidal suspension is most easily determined using static light scattering. We use a detector mounted on the arm of a goniometer to measure the angular dependence of the scattered intensity, or $I(q)$, for each of the samples studied with Brillouin scattering. We also use a very dilute sample of colloids, with $\phi$ on the order of $10^{-4}$, to measure the angular dependence of the static light scattering from isolated spheres, thereby determining their form factor, $P(q)$. The static structure factor is then determined by calculating $S(q)=I(q) / P(q)$. In this fashion, we can verify that the static structure factor is well described by that for hard spheres at the same volume fraction, as has been shown previously for these PMMA samples [11]. For the small spheres, we can clearly vary the scattering angle to attain scattering vectors smaller than the peak at $q d \approx 2 \pi$. At these small scattering vectors, the wavelengths of sound is larger than the diameter of the spheres. Moreover, we can attain scattering wave vectors near the peak of the static structure factor, where the sound wavelength is comparable to the diameter of the spheres, and also at scattering wave vectors significantly larger than the peak in the static structure factor, where the sound wavelength is much smaller than the diameter of the spheres. Thus by varying the scattering angle of our Brillouin measurements, we can determine the behavior of sound as its wavelength is varied from smaller than, to comparable to, then to greater than the diameter of the spheres.

To verify the essential properties of this system, we first acquire data at very low scattering wave vectors, where the wavelength of the sound is much greater than the diameter of the spheres. Here we expect hydrodynamic behavior, with only a single propagating longitudinal sound wave. The lowest scattering angle that we are able to attain is $\theta \approx 9^{\circ}$, corresponding to $q d \approx 0.8$, although the spectra were of poor quality at this low a scattering angle. The quality of the data improves significantly as the scattering angle is increased, and in Fig. 1 we show a series of Brillouin spectra collected for several different volume fractions, $\phi=0.16,0.38$, and 0.51 , using the small spheres, with excitations at $6471 \AA$ and a scattering angle of $\theta=16^{\circ}$, corresponding to $q d=1.7$. For all volume fractions, we observe only a single mode, as reflected by a single pair of Brillouin peaks. For the lowest volume fraction, $\phi=0.16$, the Brillouin peaks are virtually unchanged, both in shape and intensi-

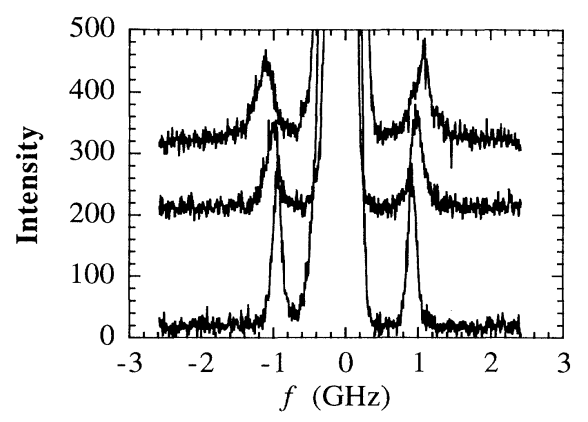

FIG. 1. Brillouin spectra for three different volume fractions, $\phi=0.16,0.38$, and 0.51, of 340-nm-diam PMMA spheres measured at $q d \approx 1.7$, in the hydrodynamic, or long-wavelength regime of propagation of acoustic waves.

ty, from that of the pure index-matching fluid, with no spheres present. However, the Rayleigh peak is increased in intensity by several orders of magnitude, reflecting the additional static scattering from the spheres, despite the good index matching. As $\phi$ increases, the Brillouin peaks shift to higher frequencies and become broader. This behavior is expected, as the velocity of sound in the solid of the spheres is roughly twice that in the fluid, so that the average velocity in the medium should increase.

Since the wavelength of the sound is still substantially larger than the diameter of the spheres, the dependence of the velocity on $\phi$ can be described with an effectivemedium model [12]. For these isolated spheres, we use Wood's model [5] to describe the average elastic modulus of the medium, $\beta$. Thus we have

$$
\frac{1}{\beta}=\frac{\phi}{\beta_{s}}+\frac{1-\phi}{\beta_{f}},
$$

where $\beta_{s}$ and $\beta_{f}$ are the elastic moduli of the pure indexmatching fluid and the pure PMMA, respectively. The velocity of sound is then given by

$$
v=\sqrt{\beta / \rho}
$$

where $\rho$ is the volume averaged density, given by

$$
\rho=\phi \rho_{s}+(1-\phi) \rho_{f},
$$

where again the subscripts $s$ and $f$ refer to the soid spheres and the fluid, respectively. Wood's approximation for the average velocity is exact in the limit of long wavelength [5].

All of the parameters in these expressions can be determined from independent measurements. The elastic moduli for the pure index-matching fluid and the PMMA spheres are determined by using Brillouin scattering to measure the sound velocity and then calculating the elastic moduli from Eq. (2). The sound velocity of the indexmatching fluid is measured directly. However, to measure the sound velocity in the PMMA, it is preferable to use the spheres themselves, as their properties may differ somewhat from the bulk polymer. Fortunately, as discussed later, it is possible to observe a peak in the Brillouin scattering from the larger spheres at very high scattering angles which corresponds to the longitudinal 
sound wave within the spheres themselves. At high angles, the inverse of the scattering vector is sufficiently small, and $q d$ sufficiently large, that several sound wavelengths fit within each sphere, allowing us to determine the speed of sound within the spheres. From these measurements, we obtain $v_{s}=2.29 \mathrm{~km} / \mathrm{sec}$. For the velocity of the index-matching fluid, we find $v_{f}=1.18 \mathrm{~km} / \mathrm{sec}$. We use the literature value for the density of PMMA, and calculate the density of the index-matching fluid from the known volumes of each fluid used and the literature values of their respective densities. This enables us to calculate the average velocity of sound in the medium using Eqs. (1)-(3) above. A comparison of this calculation and the data obtained at $\theta=16^{\circ}$ is shown in Fig. 2 . As can be seen, excellent agreement is obtained, confirming this description of the single longitudinal mode in the long-wavelength limit. We emphasize that there are no fitting parameters in comparing the calculated behavior with the measurements, as all quantities in Eqs. (1) and (2) are known independently. Similarly good agreement is found for the data obtained at lower scattering angles. Furthermore, much better agreement is obtained by calculating the volume fraction using the value of the core radius of the spheres rather than the radius which determines their phase behavior and includes the thickness of the grafted polymer layer. This confirms that, for sound propagation, the grafted polymer layer behaves more like the fluid than the solid.

As the scattering wave vector increases, a qualitative change is observed in the behavior of the Brillouin peaks. The frequency shift of the peaks no longer increases with $\phi$ as was the case at smaller $q$. Moreover, the width of the peaks becomes larger. For the small spheres, this change is first observed as $q$ approaches $0.009 \mathrm{~nm}^{-1}$, corresponding to a value of $q d$ around $\pi$, where the wavelength of the sound is roughly twice the diameter of the spheres. Most surprisingly, for volume fractions larger than 0.2, a new, second Brillouin peak is clearly observed at a higher frequency. The intensity of this second peak is much weaker than that of the first peak for $q d$ around $\pi$. Similar behavior is observed for the larger spheres, but at

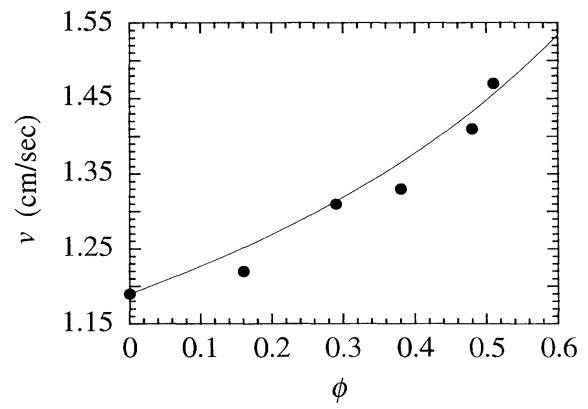

FIG. 2. The volume fraction dependence of the sound velocity in the smaller spheres, measured at small angle, corresponding to $q d \approx 1.7$, in the hydrodynamic regime for the propagation of acoustic waves. The solid line is the calculation using the effective-medium theory. The measured values of the sound velocities in the pure phases are used in the calculation. smaller angles, so that $q d$ is also roughly $\pi$. In addition, for the larger spheres, the second peak is apparently observed only for somewhat higher volume fractions than is the case for the small spheres. An example of the spectra from the larger spheres illustrating the onset of the second peak for volume fractions of $\phi=0.10,0.36$, and 0.57 is shown in Fig. 3. A weak peak is clearly observed for $\phi=0.57$, and is also present, albeit less distinct, for $\phi=0.36$. By contrast, for the $\phi=0.10$, there is not even a hint of a second peak.

As the scattering wave vector increases still further, these changes become more pronounced. Both Brillouin modes are clearly resolved, and are of roughly equal intensity by the time $q d \approx 2 \pi$. An example of the spectra obtained for three different volume fractions of the small spheres when $q d=2.8 \pi$, corresponding to $q=0.26$ $\mathrm{nm}^{-1}$, is shown in Fig. 4. At the two higher volume fractions, $\phi=0.51$ and 0.38 , the two Brillouin peaks can each be clearly distinguished. At the lowest volume fraction, $\phi=0.16$, the single peak observed appears to be somewhat asymmetric, suggesting that it may be composed of two peaks that are not completely resolved. Furthermore, as the volume fraction increases, the frequency shift between the two modes increases. This occurs because the frequency of the lower-frequency mode decreases with increasing volume fraction, while that of the higher-frequency mode increases with increasing volume fraction. In all cases, both peaks correspond to longitudinal modes, as confirmed by the absence of any peaks in the depolarized spectra. In Brillouin scattering, longitudinal acoustic modes couple only to polarized scattering, while transverse modes can couple to depolarized scattering as well [13].

Finally, to investigate the behavior at the largest possible values of $q d$, we measure the spectra of the larger spheres at high angles, and change the spacing of the Fabry-Pérot interferometer to increase the free spectral range, allowing us to resolve higher frequencies. A typical spectrum for $\phi=0.57$, obtained at a scattering angle of $171^{\circ}$, corresponding to $q d \approx 23.9 \mathrm{~nm}^{-1}$, is shown in Fig. 5. Now, instead of two peaks, three Brillouin peaks are resolved. Here, $q d$ is so large that we can resolve the Brillouin scattering independently in each of the indivi-

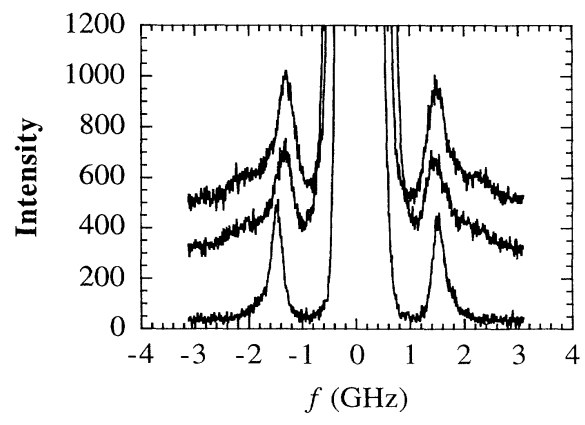

FIG. 3. Brillouin spectra for three different volume fractions, $\phi=0.10,0.36$, and 0.57 , of the larger spheres with $d=650 \mathrm{~nm}$, for $q d \approx \pi$. The second mode is barely discernible in the spectrum for the highest volume fraction. 


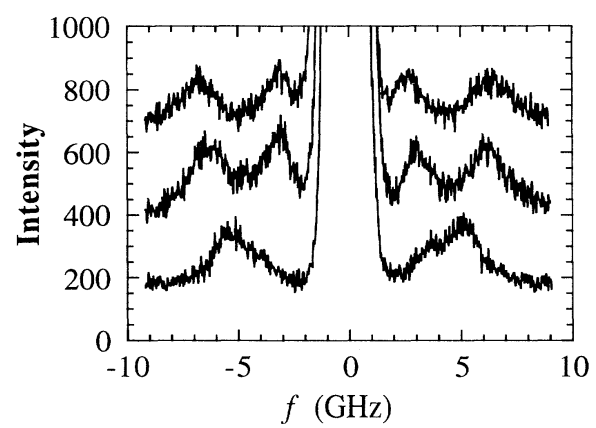

FIG. 4. Brillouin spectra for three different volume fractions, $\phi=0.16,0.38$, and 0.51, of 340-nm-diam PMMA spheres measured at $q d \approx 2.8 \pi$. Two distinct modes are clearly observable for the two higher volume fractions.

dual phases. In particular, we can resolve the scattering from within the solid spheres themselves. This results in the highest-frequency mode. The velocity of this mode, determined from the average of several measurements of this spectrum, is $v \approx 2.29 \mathrm{~km} / \mathrm{sec}$. This provides an experimental measure of the speed of sound in the solid PMMA spheres, which was used in the effective-medium theory that describes the data at small $q d$. This value is slightly lower than that of bulk PMMA (Lucite) which is $2.7 \mathrm{~km} / \mathrm{sec}$ [14], presumably reflecting the difference in the material that makes up the spheres.

We summarize the behavior of the sound propagation by plotting the dispersion relations. In Fig. 6, we plot the behavior for three different volume fractions of the small spheres, $\phi=0.16,0.38$, and 0.51 . We note that these core volume fractions correspond to effective phase volume fractions, which includes the thickness of the grafted polymer layer, of $0.21,0.49$, and 0.64 , respectively. Thus the highest volume fraction represents the maximum

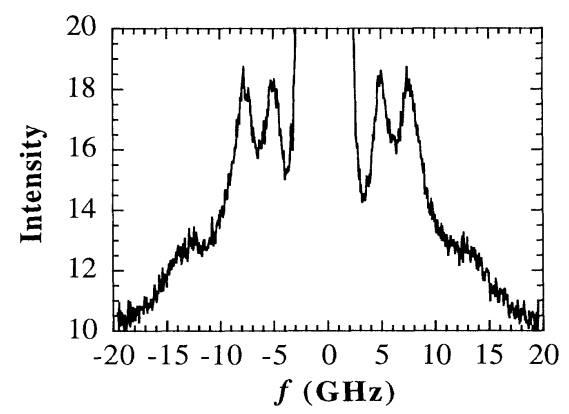

FIG. 5. Brillouin spectra at very high $q d$, obtained with the larger spheres at an angle of $171^{\circ}$ corresponding to $q d \approx 23.9$. Three distinct modes are now observed. The highest-frequency mode, which is observed as a shoulder on the tail of the other two, corresponds to sound propagation in the pure solid, while the middle mode corresponds to sound propagation in the pure index-matching fluid. The lowest-frequency mode corresponds to a Stoneley wave propagating along a nearly flat interface. At these high values of $q d$, the wavelength is sufficiently small for the sound modes to be resolved within the pure phases, and for the interface between them to appear nearly flat.

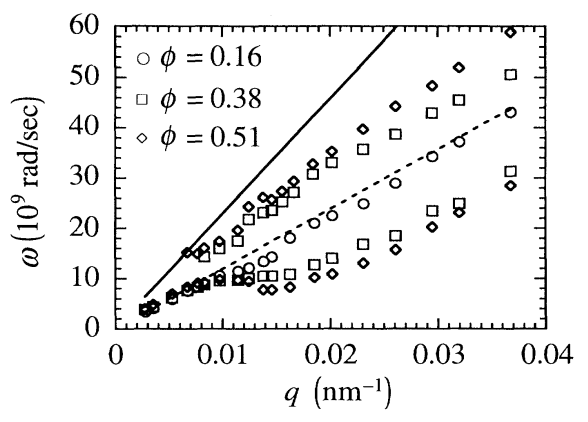

FIG. 6. The dispersion curves for the longitudinal acoustic modes for three different volume fractions, $\phi=0.16,0.38$, and 0.51 , of the $340-\mathrm{nm}$-diam spheres. The lowest volume fraction exhibits only a single mode, while the two other volume fractions exhibit two modes above $q \approx 0.008 \mathrm{~nm}^{-1}$. The splitting between the modes increases with volume fraction. The solid line represents the dispersion curve for the pure PMMA while the dashed line represents the dispersion curve for the pure index-matching fluid.

packing that can be achieved for randomly packed hard spheres. Moreover, we again emphasize that the solid cores of the spheres never touch their neighbors, even at the highest volume fraction, because of the stabilizing layer of grated polymer. For comparison, we also show in Fig. 6 the linear dispersion curves expected for the longitudinal sound waves of the pure materials that comprise the suspensions of hard spheres. The solid line corresponds to the solid PMMA while the dashed line corresponds to the index-matching fluid. The sound velocities used to plot these dispersion curves for the pure phases are obtained experimentally: The velocity in the pure index-matching fluid is measured directly from the Brillouin peaks in the pure fluid, while that in the PMMA spheres is determined from Brillouin scattering from the large spheres at very high scattering angle.

There are several remarkable features in the dispersion curves for sound propagation in hard spheres, shown in Fig. 6. At low $q$, only a single mode is observed, for all $\phi$, with linear dispersion as $q$ goes to zero. The frequencies of this mode increase with increasing volume fraction of the PMMA. However, as $q$ increases further, the dispersion curves for all three volume fractions begin to flatten and the frequency of the mode for the higher volume fractions actually drops below that of the lower volume fractions. For $\phi$ above 0.16, the second, higher-frequency mode appears at $q \approx 0.009 \mathrm{~nm}^{-1}$, corresponding to $q d \approx \pi$. This mode persists as $q$ increases. Furthermore, the splitting between the two modes increases with increasing volume fraction. By contrast, at the lowest volume fraction shown, $\phi=0.16$, the measured frequency of the single mode observed is virtually unchanged from that of the pure index-matching fluid.

It is the behavior of the higher volume fractions of the hard-sphere suspensions that is the most surprising. For these, two distinct modes are resolved for all $q$ above $0.009 \mathrm{~nm}^{-1}$, corresponding to $q d$ above $\pi$. The frequency of the higher mode always lies between the frequencies for the pure liquid and the pure solid phases. In addition, 
its frequency increases with increasing volume fraction. This behavior is characteristic for longitudinal sound propagation in a composite material comprised of two phases, one with a larger sound velocity than the other. By contrast, the behavior of the lower-frequency mode is much more unusual, and unexpected. Its frequency lies below that of the lowest frequency for the velocity in any of the pure phases that make up the medium. Moreover, its frequency decreases with increasing volume fraction of the solid phase, which adds more material with a higher velocity of sound to the composite material. Finally, the frequencies for both modes, for all three volume fractions, soften around the peak in the static structure factor, which occurs around $q \approx 0.015 \mathrm{~nm}^{-1}$, corresponding to $q d \approx 2 \pi$. The softening of the lower-frequency mode is considerably more pronounced, and the degree of softening increases with increasing volume fraction.

To investigate the behavior of the sound propagation at even larger values of $q d$, and to compare the behavior for different sizes spheres, we also measure the dispersion curves for the larger spheres, and plot them in Fig. 7 for three different volume fractions, $\phi=0.10,0.36$, and 0.57 . The overall trends of these data are the same as for the small spheres. At the very lowest values of $q$, only a single mode is observed, while for all values above $q \approx 0.005$ $\mathrm{nm}^{-1}$, two distinct modes are again observed. Again, the frequency of the higher mode lies between that of the pure index-matching fluid and the pure solid, while that of the lower mode lies below those of both the pure phases that comprise the suspension. Moreover, the splitting between the two modes again increases with increasing volume fraction.

To better compare the behaviors of the two different sized spheres, we scale the two sets of data together and plot the results in Fig. 8. The scaling is accomplished by multiplying each axis by the diameter of the sphere, $d$. Thus, on the horizontal axis we plot $q d$, which is a dimensionless quantity, while on the vertical axis, we plot $\omega d$, which has units of velocity. The vertical axis could

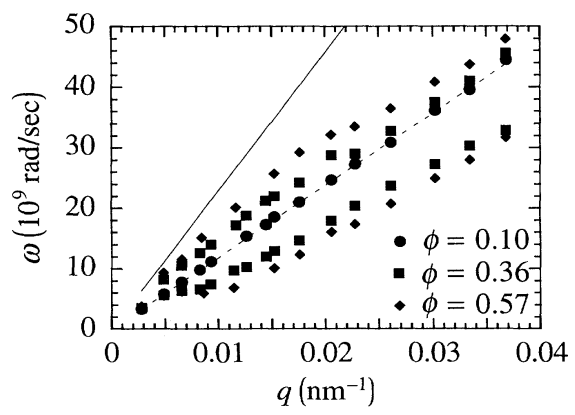

FIG. 7. The dispersion curves for the longitudinal acoustic modes for three different volume fractions, $\phi=0.10,0.36$, and 0.57 , of the larger spheres with $d=650 \mathrm{~nm}$. The lowest volume fraction exhibits only a single mode, while the two other volume fractions exhibit two modes above $q \approx 0.005 \mathrm{~nm}^{-1}$. The splitting between the modes increases with volume fraction. The solid line represents the dispersion curve for the pure PMMA while the dashed line represents the dispersion curve for the pure index-matching fluid.

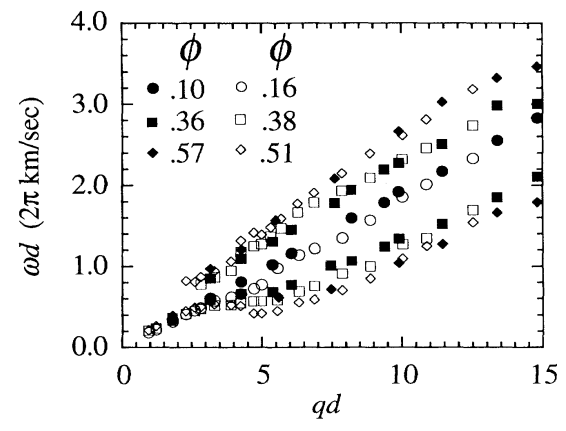

FIG. 8. Scaled dispersion curves for the 340- and 650-nmdiam spheres for different volume fractions. The solid symbols refer to the larger spheres while the open symbols refer to the smaller spheres.

also be made dimensionless by normalizing by velocity. This scaling ensures that the dispersion curves of the pure phases exhibit the proper scaling behavior. We limit the plot to the lower values of $q d$ for which overlapping data are available. In Fig. 8 , the solid points represent the data of the larger spheres, with $d=650 \mathrm{~nm}$, while the open points represent the data of the smaller spheres, with $d=340 \mathrm{~nm}$. As expected the scaled data for the two sphere sizes do exhibit the same trends: the splitting of the two modes occurs at the same point, $q d \approx \pi$, for both data sets, and both sets of data exhibit a softening around $q d \approx 2 \pi$. However, the scaling of the data is not exact. The degree of softening of the modes at $q d \approx 2 \pi$ is substantially less pronounced for the larger spheres than for the smaller spheres. Furthermore, the splitting between the normalized frequencies of the two modes is consistently less pronounced for the larger spheres than for the smaller spheres. Thus the high-frequency mode of the larger spheres always has a lower normalized frequency for a given value of $q d$ and $\phi$ than that of the smaller spheres. Similarly, the low-frequency mode of the larger spheres always has a larger normalized frequency for a given value of $q d$ and $\phi$ than that of the smaller spheres. Consequently, a larger volume fraction of spheres is required for the larger spheres to clearly resolve the two modes.

In addition to the inexact scaling of the modes for the two sizes of spheres, the behavior of the larger spheres also exhibits clear trends as $q d$ increases, which cannot be seen for the smaller spheres because of the limits in the values of $q d$ that can be achieved. To illustrate these trends more clearly, in Fig. 9 we plot the phase velocities, $v=\omega / q$, of the two modes for several volume fractions of the larger spheres. The phase velocity for the single mode observed for the lowest volume fraction, $\phi=0.10$, is almost indistinguishable from that of the pure indexmatching fluid, and exhibits little dispersion for all the wave vectors probed, as shown by the diamonds in Fig. 9. By contrast, the dispersion in the phase velocities of both the modes observed for the higher volume fractions is apparent. However, at large values of $q d$, the velocities of both modes appear to approach asymptotic values, which also appear to be independent of the volume fraction. 


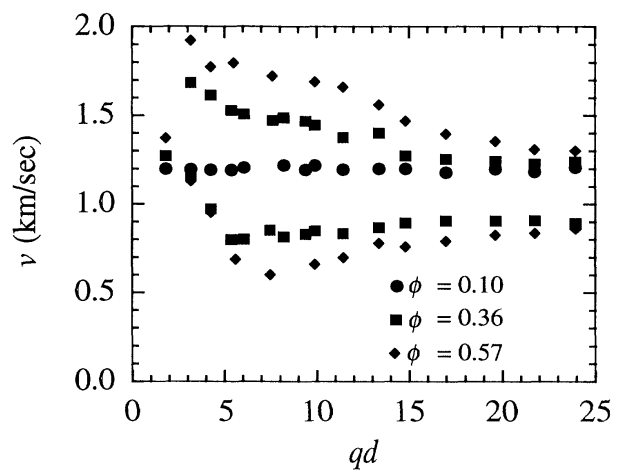

FIG. 9. The phase velocities for three different volume fractions of the 650-nm-diam spheres, plotted as a function of $q d$. The velocity of the single mode present for the lowest volume fraction is nearly indistinguishable from that of the liquid, 1.18 $\mathrm{km} / \mathrm{sec}$. The velocity of the higher-frequency mode reaches a peak around $q d \approx \pi$, then decreases as $q d$ increases, ultimately asymptotically approaching that of the pure fluid for both volume fractions. The velocity of the lower-frequency mode decreases until $q d \approx 2 \pi$, then increases, asymptotically approaching a value of $0.86 \mathrm{~km} / \mathrm{sec}$ for both volume fractions, corresponding to the velocity of a Stoneley wave at a flat interface between solid PMMA and the index-matching fluid.

While the velocity of the faster mode is considerably greater than that of the fluid at lower values of $q d$, at larger values, it decreases and appears to asymptotically approach the velocity of the pure index-matching fluid. In addition, the velocity of the slower mode displays a softening around $q d \approx 2 \pi$, but as $q d$ increases, the velocity appears to approach a constant value that is well below that of the index-matching fluid.

Finally, we also measure the widths of the Brillouin modes, which we express in terms of a quality factor, $Q=\omega / \delta \omega$, where the width $\delta \omega$ is the measured full width at half maximum of the mode. The quality factor reflects the number of wavelengths the sound mode propagates before it is damped. In Fig. 10 we plot the quality factors measured for the smaller spheres as a function of $q d$. As

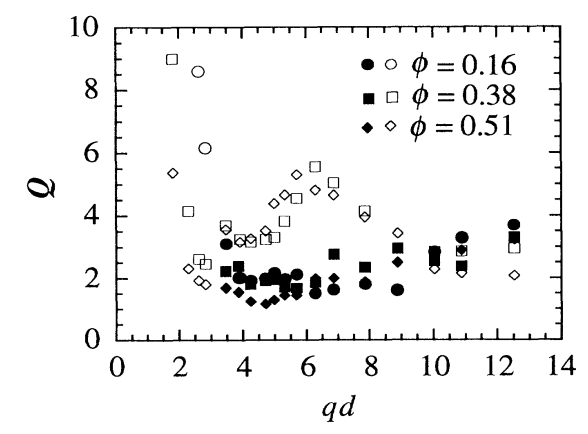

FIG. 10. The quality factors, $Q=\omega / \delta \omega$, for the two modes for three different volume fractions, $\phi=0.16,0.38$, and 0.51 , of the 340-nm-diam spheres, plotted as a function of $q d$. The $Q$ of the higher-frequency mode exhibits a pronounced peak around $q d \approx 2 \pi$. $q d$ approaches zero, the lower-frequency mode is well defined, as indicated by its large values of $Q$. However, as $q d$ increases above about $\pi$, the value of $Q$ for the lowerfrequency mode has decreased to a value of only about 2 , where it remains for all higher values of $q d$. By contrast, the $Q$ of the higher-frequency mode exhibits a pronounced resonance around $q d \sim 2 \pi$, reflecting a decrease in the damping of the mode. We emphasize that the measured quality factors reflect the damping of the acoustic modes in the composite medium of the spheres and fluid. The $Q$ for the acoustic modes in either of the pure media is significantly higher. The increased damping of the sound modes in the composite medium results from the increased scattering of the acoustic excitations by the spheres, rather than from viscous dissipation. The behavior of $Q$ for the larger spheres is consistent with that shown in Fig. 10 for the smaller spheres, but the resonance at $q d \sim 2 \pi$ is not as pronounced, since the resolution in $q d$ is not as high.

\section{DISCUSSION}

The most surprising feature of the dispersion curves for hard-sphere colloids is the existence of two distinct modes. For these systems, the continuous phase is a fluid, which has no long-range rigidity, and thus cannot support shear. Therefore only a single longitudinal mode is expected. In fact, at very long wavelengths, this can be shown rigorously to be the case [5]. Previous experiments measuring sound propagation in suspensions of hard spheres also found only a single mode [4]. These experiments probed spheres made of glass rather than polymer, and used ultrasonic techniques to measure the actual propagation of sound through the beads. They were limited to wavelengths much larger than the sphere size, but it was long assumed that the observation of the existence of only a single mode of propagating sound would persist to shorter wavelengths as well. This hypothesis was supported by theoretical treatments using a multiple scattering formalism to calculate the sound propagation through suspensions of solid spheres.

An alternate possible explanation of these observations is by analogy to the theoretical proposals of Biot $[15,16]$. $\mathrm{He}$ considered the propagation of sound in a porous medium at wavelengths much larger than any characteristic length scales of the medium, and at high frequencies. The characteristic frequency required is determined by the viscous penetration length, defined as $l=\sqrt{\rho / \eta \omega}$, where $\rho$ is the density of the solid and $\eta$ is the viscosity of the fluid. This is the length scale of viscous coupling of transverse waves from the solid into the fluid. When the frequency is sufficiently high that $l$ is less than the pore size, the Biot theory applies. Then, the solid and the fluid become decoupled, with one sound mode propagating through the solid phase and a second, new mode propagating through the fluid phase. This new slow mode is a wave that propagates predominantly in the fluid, but its velocity is slower than that in the pure fluid phase because of the tortuosity of its path. The veracity of Biot's prediction has been confirmed, both for sound propagation in some porous media structures [17] and for sound 
propagation through super fluid helium in a porous medium [18]. At the frequencies used here, the viscous penetration length is on the order of $1 \mu \mathrm{m}$ or less. This can be less than the spacing between the spheres at the lower volume fraction, although at high $\phi$, the typical spacing between the surface of the spheres is smaller than l. However, for Biot's theory to apply, the solid phase of the porous medium must be a contiguous, rigid structure and the solid and fluid must form interconnecting phases. This is not the case for a suspension of hard-sphere colloids. Because of the grafted polymer stabilizing layer, the solid spheres are not interconnected, even at the highest volume fractions studied. Furthermore, even if the solid phase was completely interconnected, its tortuosity is very nearly unity $[19,20]$, so the decrease in the sound velocity of the lower-frequency mode cannot be ascribed to a Biot slow wave. Thus we reject the Biot approach in describing these results.

Instead, we suggest an alternate physical picture of the two modes. We consider the nature of each mode individually. The higher-frequency mode has the dependence on volume fraction expected for an acoustic excitation propagating through a composite medium: the frequency increases as the volume fraction of solid increases. Since the velocity of sound is much greater in the pure solid than in the pure fluid, the frequency of an acoustic excitation propagating through the two media should also increase. Furthermore, the frequency of this mode lies between those of the two pure materials. Thus we attribute the higher-frequency mode to an acoustic excitation that propagates through both the fluid and the solid. Moreover, since the single excitation observed at low $q d$ also has the same dependence on volume fraction, and is well described by an effective medium model which assumes that it propagates through the two materials, it too must be associated with the acoustic excitation propagating through the liquid and the solid spheres.

The nature of the propagation of this mode is strongly influenced by the presence of the spheres. While the index of refraction of the fluid and the solid are very nearly matched, eliminating any strong scattering of light from the system, this is not the case for sound. There is a large impedance mismatch for sound to propagate from one material to the other, and as a result, the sound waves are strongly scattered from the interfaces. The consequence of this is most clearly observed by examining the dispersion curve for this mode for a single volume fraction. As an example, we plot, in Fig. 11, the dispersion curve for the higher-frequency mode for the smaller spheres with $\phi=0.38$. Two gaps in the dispersion curve are clearly evident at frequencies of $\omega \approx 10 \times 10^{9}$ and $20 \times 10^{9}$ $\mathrm{rad} / \mathrm{sec}$, corresponding to frequencies where the excitation does not propagate. Moreover, the dispersion curve becomes flat on either side of the gaps. The frequencies of these gaps correspond to the frequencies calculated for the peaks in the scattering amplitude of a longitudinal wave incident on a single sphere immersed in a fluid, which are shown by the two arrows. Thus when the scattering from the isolated spheres becomes very large, the wave can no longer propagate, and gaps develop in the dispersion curve. The large increase in the scattering

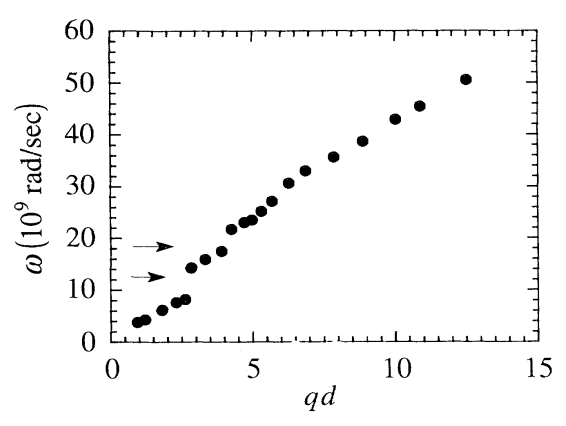

FIG. 11. The dispersion curve of the high-frequency mode for the $340-\mathrm{nm}$-diam spheres at $\phi=0.38$. The arrows correspond to the frequencies of the peaks of the scattering amplitudes of longitudinal acoustic waves incident on a single, isolated sphere immersed in a fluid.

from the individual spheres results from the excitation of internal resonances within the spheres [21,22]. There are a large number of these resonances at higher frequencies, with the two gaps observed corresponding to the two lowest-frequency resonances. Gaps in the dispersion curve at higher frequencies may also be discernible, but not as clearly. While sound at these frequencies cannot propagate with the wave vectors corresponding to this mode, it can propagate at the same frequency, but at another wave vector. This is the lower-frequency mode.

The lower-frequency mode must have a different physical origin. It propagates at a frequency lower than that of either of the two pure phases. Moreover, its velocity decreases as the volume fraction of solids increases. This slow velocity is the key to the origin of the mode. The only mode that can propagate in a system comprised of a solid and a fluid at a velocity that is slower than the longitudinal velocities in both materials is a Stoneley wave [9]. This is a propagating wave confined to the interface between a solid and a fluid. It is analogous to a Rayleigh wave, which is a propagating wave confined to the interface of solid and vacuum. The existence of a Stoneley wave requires a shear modulus in the solid. It consists of both longitudinal and transverse polarizations in the solid and a purely longitudinal component in the fluid. The magnitude of the oscillation decays exponentially away from both of the surfaces, ensuring the confinement of the excitation to the interface. While a Stoneley wave is a solution to the elastic equations that is confined to a flat interface, the excitation here is initially observed when the wavelength of the sound is less than the size of a single sphere. Moreover, from the width of the Brillouin peaks, we can ascertain that the excitation propagates over length scales larger than a single sphere. Thus this excitation must propagate coherently from sphere to sphere. This presumably occurs through the exponentially decaying portions of the excitation in the fluid. If another sphere is nearby, the excitation can hop between the two spheres. This would account for the fact that this new mode is only observed when the volume fraction of the spheres is relatively large, ensuring that there are neighboring spheres close by. Moreover, this would account for the very unusual dependence of the velocity on 
volume fraction. At higher volume fractions, there are more interfaces relative to the amount of fluid. The velocity of the excitation on the interfaces is lower than in the fluid, thus leading to the measured decrease in the velocity as the volume fraction of spheres increases.

Support for our interpretation comes from the plot of the phase velocities measured for the larger spheres, shown in Fig. 9. The velocity of the single mode observed for the lowest volume fraction is almost indistinguishable from that of the index-matching fluid, at 1.18 $\mathrm{km} / \mathrm{sec}$. The velocity of the lower-frequency mode is initially quite slow, and decreases near $q d \approx 2 \pi$. However, as $q d$ increases to much larger values, the velocity increases somewhat, and then approaches an asymptotic value, which is independent of volume fraction. At these large values of $q d$, the interface appears to be nearly flat on the scale of a wavelength. The velocity of this mode measured at high $q d$ is $0.86 \mathrm{~km} / \mathrm{sec}$, which corresponds directly to the velocity calculated for a Stoneley wave at a flat interface between PMMA and the index-matching fluid. By contrast, the velocity of the higher-frequency mode seems to decrease and asymptotically approach the velocity of the index-matching fluid for all volume fractions of spheres. This is again consistent with an effective-medium picture for this excitation. At these very large values of $q d$, it is possible to fit several wavelengths of the excitation in either a single sphere or in the fluid between the spheres. Thus it is possible to detect the excitations within each of the pure materials that comprise the suspension. The higher-frequency excitation therefore corresponds to the longitudinal sound wave within the pure fluid. These observations lend strong support to our interpretation of the results.

\section{THEORY}

The experimental results described above represent a major challenge to theory since their explanation requires the treatment of wave propagation in a strongly scattering medium, where the wavelength is comparable to the size of the inhomogeneities. In order to achieve this goal, a generalized coherent-potential-approximation (CPA) approach is developed for the identification of quasimodes and the calculation of their dispersion relations. The basic principle of this approach is simple and may be best understood from the CPA conditions. For CPA, if we let $G$ denote the exact Green's function for an acoustic wave in a random medium, then,

$$
G=G_{0}+G_{0} T G_{0},
$$

where $G_{0}$ is the Green's function for a homogeneous effective medium and $T$ denotes the exact scattering operator, including all the multiple scattering among the spheres. By expressing $G_{0}=\left(p^{2}-q^{2}\right)^{-1}$, where $q=\omega / c_{m}, c_{m}$ being the effective medium speed and $p$ the Fourier transform variable that is conjugate to the spatial variable, the CPA condition for Eq. (4) is $\langle T\rangle=0$ through the adjustment of $q$, where the angular brackets denote configuration average. When that happens, $\langle G\rangle=G_{0}$, and $q$ is identified as the wave vector of the excitation. Since $\langle T\rangle \approx n t$ in the weak scattering limit, where $t$ is the single-scatterer forward scattering amplitude, $n$ the volume concentration of the scatterer, $\langle T\rangle=0$ is the equivalent to $t=0$, which means the CPA condition is consistent with requiring the forward scattering amplitude to vanish for a single scatterer embedded in an effective medium. In other words, when the scattering vanishes for a single scatterer, it also vanishes for the whole medium on average, and this is precisely the condition for the existence of a coherent mode.

For the generalized CPA condition, instead of requiring $\langle T\rangle=0$, we look for minima of $\langle T\rangle$. The fact that the scattering now does not vanish on average means that the excitation must be a quasimode. However, since at minima the scattering may still be weak, we may approximate $\langle G\rangle$ by

$$
\langle G\rangle \approx \frac{1}{p^{2}-q^{2}-n t} .
$$

The minima of $\langle T\rangle$ may thus be identified by the maxima of density of states (DOS), evaluated as $(1 / \pi) \operatorname{Im}\langle G\rangle$ under the condition of elastic scattering $(p=q)$, so that $\langle G\rangle=-(n t)^{-1}$. The maxima of DOS therefore correspond directly to the minima in scattering, which, in turn, give the best condition for the existence of a quasimode since less scattering means the wave can coherently propagate over a longer distance. In this approach we also note that the minima in $\langle T\rangle$ are also the places where $\langle T\rangle \approx n t$ is the best approximation. Therefore the maxima of DOS are precisely the places where this approach is most accurate.

In order to implement this approach, it is necessary to calculate the $t$ for a single solid particle. In a colloidal suspension, each spherical solid particle is enveloped by fluid. This microgeometry is to be contrasted with the alternative possibilities in which the particles cluster and touch. To carry out Green's function calculations, this strong short-range correlation between the solid and fluid phases must be taken into account. For this purpose, we consider as the basic scattering unit a coated sphere, where the fluid coating thickness $s$ is determined by the solid particle concentration $\phi=d^{3} /(d+2 s)^{3}$.

To calculate the Green's function for a single coated sphere embedded in an effective medium, we start from the elastic wave equation for each of the three homogeneous regions,

$$
\frac{\partial^{2} \mathbf{u}}{\partial t^{2}}=c_{t}^{2} \nabla^{2} \mathbf{u}+\left(c_{p}^{2}-c_{t}^{2}\right) \nabla(\nabla \cdot \mathbf{u})
$$

where $\mathbf{u}$ is the displacement, $c_{p}$ is the longitudinal and $c_{t}$ the shear wave velocities. For the fluid coating and the effective medium, $c_{t}=0$. By decomposing $\mathbf{u}=-\nabla \psi$ $+\nabla \times \mathbf{A}$, with the vector potential $\mathbf{A}$ being further expressed as $\mathbf{A}=\boldsymbol{\nabla} \times \mathbf{r} \xi$, we get a pair of Helmholtz equations for $\psi$ and $\xi$. Here $\psi$ is directly proportional to the density variation, $\boldsymbol{\nabla} \cdot \mathbf{u}$. Since in Brillouin scattering the light is coupled to the thermal excitations through refractive-index changes induced by density variations, the relevant Green's function is that for measuring the $\psi$ response to a point scalar source, i.e.,

$$
\left[\nabla^{2}+\omega^{2} / c_{p}^{2}(\mathbf{r})\right] g_{\psi}\left(\mathbf{r}, \mathbf{r}^{\prime}\right)=\delta\left(\mathbf{r}-\mathbf{r}^{\prime}\right)
$$


where $\quad c_{p}(\mathbf{r})=c_{s}=2.7 \times 10^{5} \mathrm{~cm} / \mathrm{sec}$ for $r<d / 2$, $c_{p}(\mathbf{r})=c_{l}=1.2 \times 10^{5} \mathrm{~cm} / \mathrm{sec}$ for $d / 2 \leq d / 2+s$, and $c_{p}(\mathbf{r})=\omega / q$ for $r \geq s+d / 2$. For the $\xi$ field, on the other hand, we have $\left(\nabla^{2}+\omega^{2} / c_{t}^{2}\right) g_{\xi}\left(\mathbf{r}, \mathbf{r}^{\prime}\right)=0$ for $r<d / 2$, where $c_{t}=1.1 \times 10^{5} \mathrm{~cm} / \mathrm{sec}$, and $g_{\xi}=0$ otherwise. The $g_{\psi}$ and $g_{\xi}$ are coupled by the boundary conditions, i.e., continuity of normal displacement and normal stress, and tangential stress equals zero at $r=d / 2$; and the continuity of normal displacement and stress at $r=d / 2+s$. Here we have taken the fluid to be inviscid. The effect of viscosity is investigated and numerical simulations are presented elsewhere [22]. The Green's function $g_{\psi}\left(\mathbf{r}, \mathbf{r}^{\prime}\right)$ is obtained by solving three boundary value problems where the source point $\mathbf{r}^{\prime}$ is located in each of the three spatial regions (sphere, coating, and the effective medium). By Fourier transforming $g_{\psi}\left(\mathbf{r}, \mathbf{r}^{\prime}\right)$, we get $g_{\psi}\left(\mathbf{p}_{1}, \mathbf{p}_{2}, q ; \omega\right)$, where the dependence on $q$ and $\omega$ is explicitly noted. Since $g=G_{0}+G_{0} t G_{0}$, the scattering operator $t$ for a single coated sphere may be thus obtained. In accordance with the discussion above, the final result of our calculation is the density of states, $\operatorname{Im}\langle G\rangle / \pi$, evaluated with the condition of elastic scattering.

The results of such calculations have been given in Refs. [21,22]. With no adjustable parameters, the theoretical results are in excellent quantitative agreement with the data. Both the positions of the peaks of the modes, and their widths, are determined theoretically, and are in accord with the data presented here. A separate calculation shows the frequency positions of the sphere resonant scattering, indicated by the black arrows in Fig. 11, coincide remarkably well with the gaps in the dispersion relation of the high-frequency mode, and offer the key to its understanding as arising from the antiresonance conditions for the single-sphere scattering. For the low-frequency mode, on the other hand, the frequency positions of the resonant scattering coincide with peaks in the DOS. The maxima of DOS in this case arise from the interaction between neighboring spheres and the resulting split of a single resonance into two, with a scattering minimum in between. This scattering minimum thus represents the antiresonance condition of two coupled spheres. It can be picked out from our single-sphere calculations because the effective medium $q$ is treated as a variable, and as its value is scanned it effectively varies the impedance at the coating-effective-medium interface, which, from the point of view of a single sphere, represents its interaction with the neighboring spheres. A more detailed theoretical treatment of the problem, including the calculation of the multisphere model and numerical simulation, has been presented in Refs. [21,22].

\section{CONCLUSIONS}

In this paper, we have presented the results of an experimental study of the propagation of longitudinal sound through a suspension of solid spheres. We use PMMA spheres immersed in an index-matching fluid, eliminating any multiple scattering of light. This enables us to use Brillouin scattering to measure the thermally excited sound waves in the suspension. By varying the scattering angle, as well as the size of the spheres, we are able to probe the frequency of the propagating sound waves over values of $q d$ extending from about 1 to about 25. This corresponds to the variation of the wavelength of sound from values less than the diameter of the spheres to much greater than the diameter of the spheres. Unexpected results are found. When the sound wavelength is larger than the diameter of the spheres, only a single longitudinal mode is observed, as expected for a medium that has no long-range rigidity. However, when the wavelength of sound becomes comparable to the diameter of the spheres, two longitudinal modes are observed. The velocity of the higher-frequency mode lies between those of the pure liquid and pure solid phases, and it increases with increasing volume fraction of the solid, $\phi$. By contrast, the velocity of the lower-frequency mode is less than those in either of the two pure phases. Moreover, it decreases with increasing $\phi$.

We interpret the higher-frequency mode as an excitation that propagates within both the liquid and the solid phases, as expected for a composite medium. In fact, at low $q d$, the $\phi$ dependence of this mode is well described by a simple effective-medium theory. As the volume fraction of solids increases, the excitation is increasingly in the solid phase, accounting for the increased velocity. However, this excitation is strongly scattered by the presence of the spheres. As a result, gaps develop in its dispersion curve, and the group velocity of the mode approaches zero near these gaps. The gaps occur at frequencies that correspond well to the resonances in the scattering from an isolated sphere in a fluid, where the scattering is a maximum.

We interpret the lower-frequency mode as an analog of an interfacial, or Stoneley wave. This is the only mode that can propagate at a velocity slower than either those of the liquid or the solid. This excitation is a mixed longitudinal and transverse excitation in the solid and is purely longitudinal in the fluid. Its amplitude decays exponentially away from the interface in both the solid and the fluid. In addition, it exists only for an elastic solid, which can support shear modes, immersed in a fluid. Our interpretation is supported by the data at very large $q d$, where the wavelength is sufficiently small that the interface appears nearly flat to the sound waves. Then the velocity of this excitation approaches that calculated for a Stoneley wave at a flat interface between PMMA and the index-matching fluid. At smaller values of $q d$, this mode persists. When the wavelength is comparable to the diameter of the spheres, the mode must propagate between spheres through the longitudinal decaying portion of the excitation in the fluid. Thus the mode is observed only when the volume fraction of the spheres is sufficiently large, greater than $\phi \approx 0.2$. Moreover, since the velocity of the interfacial portion of the excitation is so slow, the measured velocity of the mode decreases as $\phi$, and thus the amount of interface, increases.

Finally, in this paper, we have also presented a brief summary of an extensive theoretical treatment of the propagation of sound in suspensions of solid spheres. This entailed an effective-medium approach, where the basic unit of the medium is a solid sphere coated by a lay- 
er of fluid, whose thickness is determined by the volume fraction. This basic unit is embedded within an effective medium, and the elastic wave equation is solved for each region to obtain the Green's function. This is used to calculate the density of states of phonon quasimodes. Maxima in this density of states correspond to minima in the scattering of the excitation, and thus to propagating modes. With this approach, we obtain quantitative agreement with the experimentally measured dispersion curves.

In conclusion, we have reported the detailed results of a study of sound propagation in suspensions of solid spheres. Despite the fact that sound propagation in these systems has been studied for over 100 years, the results reported here demonstrate that unexplored and interesting phenomena still exist.

\section{ACKNOWLEDGMENT}

We very gratefully acknowledge Professor Peter Pusey for many valuable discussions during the early stages of this work, and for very kindly supplying the PMMA samples.
*Present address: Physics Department, Brookhaven National Laboratory, Upton, NY 11973.

$\dagger$ Present address: Physics Department, California State University at Long Beach, Long Beach, CA 90840.

[1] Lord Rayleigh, The Theory of Sound (Dover, New York, 1945).

[2] J. J. Faran, J. Acoust. Soc. Am. 23, 405 (1951).

[3] G. T. Kuster and M. N. Toksoz, Geophysics 39, 607 (1974).

[4] L. M. Schwartz and T. J. Plona, J. Appl. Phys. 55, 3971 (1984).

[5] Z. Hashin, J. Appl. Mech. 29, 143 (1962).

[6] I. S. Graham, L. Piche, and M. Grant, Phys. Rev. Lett. 64, 3135 (1990).

[7] J. Liu, L. Ye, D. A. Weitz, and P. Sheng, Phys. Rev. Lett. 65, 2602 (1990).

[8] D. A. Weitz, J. Liu, L. Ye, and P. Sheng, in Physical Phenomena in Granular Materials, edited by G. C. Cody, T. Geballe, and P. Sheng (Materials Research Society, Pittsburgh, 1990), Vol. 195, p. 93.

[9] L. M. Brekhovskikh, Waves in Layered Media (Academic, New York, 1980).

[10] P. N. Pusey and W. van Megan, Nature (London) 320, 340 (1986).
[11] P. N. Pusey, in Liquids, Freezing and Glass Transition, edited by J. P. Hansen, D. Levesque, and J. Zinn-Justin (Elsevier, New York, 1991), p. 765.

[12] P. Sheng, in Homogenization and Effective Moduli of Materials and Media, edited by J. L. Ericksen, D. Kinderlehrer, R. Kohn, and J.-L. Lions (Springer-Verlag, New York, 1986), p. 196.

[13] B. J. Berne and R. Pecora, Dynamic Light Scattering: with Applications to Chemistry, Biology and Physics (Wiley, New York, 1976).

[14] CRC Handbook of Chemistry and Physics, edited by R. C. Weast (CRC, Boca Raton, 1985).

[15] M. A. Biot, J. Acoust. Soc. Am. 28, 168 (1956).

[16] M. A. Biot, J. Acoust. Soc. Am. 28, 179 (1956).

[17] T. J. Plona, Appl. Phys. Lett. 36, 259 (1980).

[18] J. Beamish, A. Hikata, and C. Elbaum, Phys. Rev. B 27, 5848 (1983).

[19] M. Y. Zhou and P. Sheng, Phys. Rev. B 39, 12027 (1989).

[20] P. Sheng and M. Y. Zhou, Phys. Rev. Lett. 61, 1591 (1988).

[21] X. D. Jing, P. Sheng, and M. Y. Zhou, Phys. Rev. Lett. 66, 1240 (1991).

[22] X. D. Jing, P. Sheng, and M. Y. Zhou, Phys. Rev. A 46, 6513 (1992). 